\title{
A PRACTICAL APPROACH FOR MODELLING THE ELECTROMAGNETIC RADAR SIGNATURE OF BAREFACED TERRAIN FOR REMOTE SENSING
}

\author{
M. EZEOKE ${ }^{1,2}$, K. TONG ${ }^{1} \&$ S. SHI ${ }^{3}$ \\ ${ }^{1}$ Department of Electronic \& Electrical Engineering, University College London, UK. \\ ${ }^{2}$ National Space Research and Development Agency, Obasanjo Space Center, Abuja. \\ ${ }^{3}$ Department of Civil Engineering, University College London, UK.
}

\begin{abstract}
A practical approach is proposed and used to investigate the electromagnetic (EM) signature of barefaced terrain using 3D computer electromagnetic models (CEM). Six barefaced terrain types with different electrical, physical and chemical properties were investigated. They comprise homogeneous and heterogeneous terrain. The approach developed CEMs in software using reflectance spectroscopy and dielectric permittivity data. EM signature models of the barefaced terrain are based on finite integration technique (FIT) solvers. The developed technique and models are valid for diverse materials under test including unconventional petroleum resources like shale rock and oil sands. The remote sensing of terrain from airborne or satellite synthetic aperture radar requires a prior determination of the EM signature for accurate classification. The implementation of our new method combined empirical measurements and FIT in three steps. Geochemical properties determined using reflectance spectroscopy in the mid-infrared region $(2.5-25 \mu \mathrm{m})$ identified the presence of bitumen, clay and moisture in Nigerian oil sands while reststrahlen effects were observed in beach sand compared with gravel and pebble. Also new information on both real, $\varepsilon_{r}^{\prime}$ and imaginary, $\varepsilon_{r}^{\prime}$ permittivity of terrain was experimentally obtained for frequency varying from 1 to $11 \mathrm{GHz}$. After post-processing, the results differed from expectation of complex refractive index method for petrophysics although adequate Kramers-Krönig correlation between measured real, $\varepsilon_{r}^{\prime}$ and imaginary, $\varepsilon_{r}^{\prime}$ permittivity data was exhibited. Our approach uses the results to improve the CEMs for superior EM signature determination. An application of our new technique to land degradation monitoring using radar is also presented.

Keywords: Barefaced terrain, complex refractive index method, computer electromagnetic model, dielectric permittivity, finite integration technique, oil sand, radar, radar signature, remote sensing, synthetic aperture radar, unconventional petroleum exploration.
\end{abstract}

\section{INTRODUCTION}

Remote sensing is the ability to detect information about an area, target or phenomenon by using a detecting sensor that is not in physical contact with the said area, target or phenomenon. Lillesand et al., [1] classically considered the human eye as a sensor because it collects data in the visible electromagnetic (EM) spectrum for analysis by the brain. Computer electromagnetic modelling (CEM) has varied applications in several fields of remote sensing including meteorology, medical imaging, military reconnaissance and mining amongst others. There are two types of sensors. Passive sensors like the human eye take advantage of naturally occurring radiation such as sunlight interacting with the object but active sensors such as radio detection and ranging (RADAR), emit their own signal, detect the returned radiation from the target and then process it. This means active sensors can be configured to highlight target objects in a best case manner. Radar sensors may be ground-mounted, air or space borne. They operate in the microwave region of the EM spectrum giving them all weather capability including day and night imaging. If mounted on an aircraft or satellite, they can also image remotely located areas. 
The target information is contained in the scattering coefficient $\left(\sigma^{0}\right)$. In a remote sensing radar image this shows up in the variation of image tone. Dobson et al. [2] explain that scattering coefficient $\left(\sigma^{0}\right)$ depends on geometric factors related to target surface attributes and electrical factors due to dielectric properties of soil and vegetation for specific wavelengths. In addition to wavelength (or frequency), other sensor parameters such as incident geometry, polarization and resolution affect $\left(\sigma^{0}\right)$. For farmland, Ulaby [3] identified a relationship between $\sigma^{0}$ and roughness while results from other studies: Ezeoke and Tong [4], Schaber [5], Ezeoke and Tong [6] and Oh et al. [7] showed diverse scattering relationships between sensor and terrain parameters.

The development of accurate algorithms for the solution of backscattering problems is important whether terrain characterization is performed offline or in situ onboard the air or space platform. This is usually a challenging task owing to the non-linear and non-unique properties of the problem, which cause the EM signature of terrain to overlap. This paper develops a practical approach to determine the EM radar signature of barefaced terrain using CEM. Other mathematical methods are complex and surface specific. For instance, the Kirchoff scattering model (KSM) applies to only relatively smooth surfaces while the small perturbation model (SPM) best represents slightly rougher surfaces [3]. However, our proposed method is simpler and by considering the actual intrinsic and extrinsic terrain properties has wider application to terrain classification for remote sensing.

Previously, Ezeoke et al. [8] considered a framework to develop radar models for petroleum exploration of oil sand. Here we expand the technique and verify that it can be applied more broadly to the field of remote sensing by considering other terrain including homogeneous environments. The new approach involves a three-step process:

Step 1: Experimentally obtain the geochemical signature through reflectance spectroscopy.

Step 2: Use the component distribution obtained in the first step as an initial guess before experimental measurements of dielectric permittivity.

Step 3: Incorporate dielectric permittivity data from second step in to CEMs which are then post-processed to provide EM signature.

At each step of the process, results were compared with literature. In Section 2, we briefly introduce the theory and measurement process while Section 3 outlines some experimental and modeling results. In Section 4, we discuss a practical application to land degradation monitoring during flooding and Section 5 concludes.

\section{THEORY AND PROCESS}

In EM signature classification, the main goal is the determination of the quantitative description of known scatterer(s) such as size, location, permittivity and conductivity from measurement data obtained away from the scatterer. For the numerical solution, the volume integral equation is an appropriate method because the calculation domain is an inhomogeneous closed space for most situations. In some general applications, a good approximation of the signature is useful. Several approximation techniques have been developed based on KSM, SPM or geometric optics approximations [9-10]. We developed a practical approach with universal applicability.

For our method, the electrochemical bonds indicative of the biophysical nutrients present were determined from hyperspectral reflectance data based on Fourier transform infrared 
(FTIR) spectroscopy. This was preferred to other techniques such as X-ray diffraction or microprobe analysis because of its suitability to study light elements such as carbon, oxygen, hydrogen and nitrogen also noted by Cloutis et al. [11]. These elements are possibly present in terrain. The electrical property most relevant to the radar signature was the dielectric permittivity $\varepsilon_{r}^{\prime}$ and $\varepsilon^{\prime \prime}{ }_{r}$ which was determined using a vector network analyser (VNA) and dielectric probe kit (DPK). The electrical properties vary with frequency therefore having a range of permittivity values better represent the scattering response obtained with the CEM after an algorithm was implemented to select the best fit from the data.

To verify our approach, we had to consider diverse terrain; therefore, the materials under test (MUT) covered several barefaced terrains. The types considered include beach sand, loamy farm sand, $10 \mathrm{~mm}$ pebbles, $40 \mathrm{~mm}$ gravel, hard oil sand (HOS) and viscous oil sand (VOS) shown in Fig. 1. Together they represent MUT A to F, respectively. The oil sand samples were obtained from the Agbabu oil sand reservoir in south west Nigeria and transported to UK. The reservoir is located within latitudes $6^{\circ} 35^{\prime} 16.3^{\prime \prime} \mathrm{N}$ to $6^{\circ} 37^{\prime} 13.9^{\prime \prime} \mathrm{N}$ and longitudes $4^{\circ} 49^{\prime} 29^{\prime \prime} \mathrm{E}$ to $4^{\circ} 50^{\prime} 20.7^{\prime \prime} \mathrm{E}$ but the other terrain types were acquired locally in UK.

\subsection{Spectral measurements}

\subsubsection{Hyperspectral reflectance measurement}

Reflectance spectroscopy has been used to study the energy reflected from a solid, liquid or gas at wavelengths that are small enough to recognize subtle changes in the underlying crystal structure of the MUT. Combinations and overtones of the different absorption (or transmission) bands that occur in the middle and far IR regions or even crystal field transitions can be detected using spectroscopic sensors in the ultraviolet, visible and NIR spectral regions. For our work beach sand, loamy farm sand, VOS and HOS did not undergo any preparation before the measurement. This is necessary to avoid influencing the results. To obtain

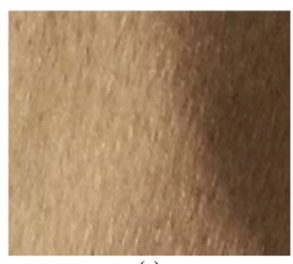

(a)

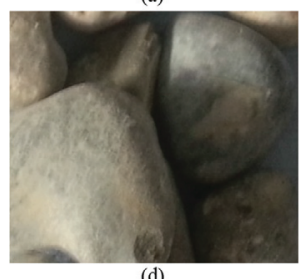

(d)

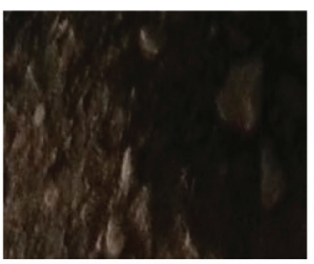

(b)

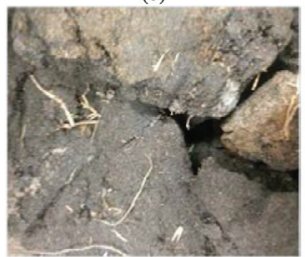

(e)

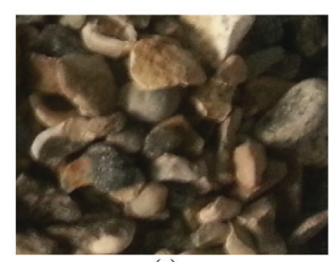

(c)

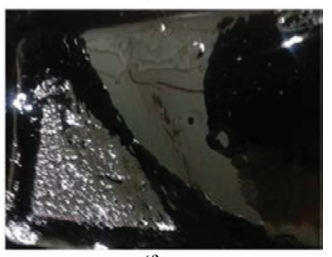

(f)

Figure 1: Barefaced terrain. First row: (a) MUT A - beach sand (b) MUT B - loamy farm sand (c) MUT C - 10-mm pebbles. Second row: (d) MUT D-40-mm gravel (e) MUT E - hard oil sand (f) MUT F - viscous oil sand. 
representative samples of pebble and gravel for spectral measurement, both pebble and gravel were soaked in deionized water and sonicated using a Branson1510 ultrasonic Sonicator. This is a useful procedure to extract representative sediments of solid and non-granular terrain for spectral identification. All the samples were characterized by FTIR. The reflectance data were acquired using a Shimadzu IRPrestige-21 spectrophotometer at the University College London, UK. The spectra were measured at $4000-400 \mathrm{~cm}^{-1}(2.5-25 \mu \mathrm{m})$; $25-100$ scans; $4 \mathrm{~cm}^{-1}$ resolution and automatic gain adjustment.

\subsubsection{Geochemical signature}

A large variety in the terrain samples means that there are several spectral absorption features that can be monitored. The geochemical signature is most useful for heterogeneous MUT such as natural gas, petroleum seeps or oil sands according to $\mathrm{Xu}$ et al. [12] and Yoon et al. [13]. We extended the applicability to homogeneous terrain by determining and then using the geochemical signature to confirm the chemical phases present. This is possible because we did not use any solvents such as tetrahydrofuran or enhancement such as potassium bromide to best compare the geochemical signatures across terrain types. The presence of coexisting anomalies due to alteration minerals and hydrocarbons means the geochemical signature was observed in the wavenumber bands, $v=600$ to $3100 \mathrm{~cm}^{-1}$ in order to best characterize IR spectra for clay and bitumen. A comparative diagnostic qualitative analysis to determine the major and minor mineral phases present per sample was performed mainly using a modification to the IR solution spectral angle mapper (SAM) classifier to match peaks and troughs.

\subsection{Electrical measurements}

\subsubsection{Dielectric permittivity measurement}

There are several methods to measure both the real $\varepsilon^{\prime}{ }_{r}$ and imaginary $\varepsilon^{\prime \prime}{ }_{r}$ of a MUT. They include the parallel plate, resonant cavity, transmission line and coaxial probe technique. The coaxial probe technique is more suited for semi-solid MUT because it increases the surface contact area and provides a relatively quicker $\varepsilon_{r}^{\prime}$ response although the $\varepsilon^{\prime \prime}{ }_{r}$ resolution is coarser than the resonant cavity method. We improved the resolution by minimizing measurement errors, recalibration and least-square fitting of several spatial measurements. Dielectric permittivity was investigated using an Agilent PNA N5227A $10 \mathrm{MHz}$ to $67 \mathrm{GHz}$ network analyser along with an Agilent 85070E DPK. Each sample was measured over a range of frequencies covering $0.5 \mathrm{GHz}$ (L-band) through C-band $(4-8 \mathrm{GHz}$ ) and up to $11 \mathrm{GHz}$ (X-band). Short, open, load and through calibrations were performed before each data acquisition to eliminate systematic measurement errors. The dielectric permittivity measurement set up for the land degradation application is shown in Fig. 2.

\subsubsection{Effect of dielectric permittivity}

Surface scattering of scalar and vector waves experience time and spatial dispersion because the permittivity of a medium depends on the frequency and wave number. For the inverse scattering problem, an accurate knowledge of the complex dielectric constant is important when construing the interaction between multilayered media [14]. To understand the effect of the dielectric permittivity, we consider an EM wave propagating from a source to a target. The time-space $(t, \mathbf{r})$ characteristic of such an EM wave is governed by the Maxwell equations, 


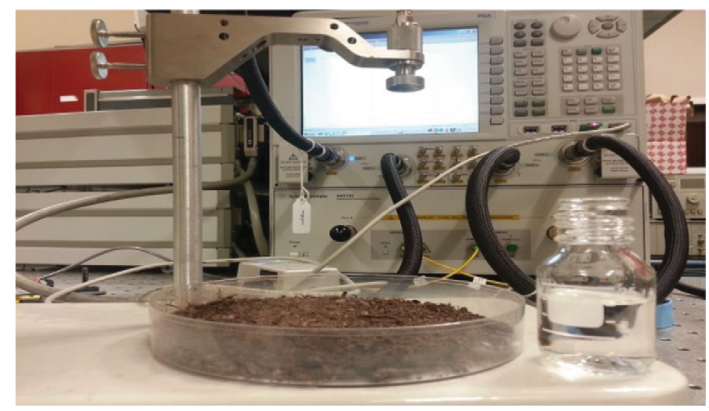

Figure 2: Generic set up for dielectric measurement of $80 \mathrm{~g}$ loamy farm sand and $20 \mathrm{~g}$ water for land degradation measurement.

which define fundamental laws for wave generation, propagation and interaction with matter. We consider a point in time $t$, and spatial location $\mathbf{r}$, with $\mathbf{r}$ being a position vector defined with respect to a coordinate system. From Ward and Hohmann [15], the Maxwell equations set for the electric and magnetic fields, $\mathbf{E}(\mathbf{r}, t)$ and $\mathbf{H}(\mathbf{r}, t)$ along with magnetic flux density and electric displacement, $\mathbf{B}(\mathbf{r}, t)$ and $\mathbf{D}(\mathbf{r}, t)$ are given by

$$
\nabla \cdot \mathbf{D}=\rho ; \nabla \times \mathbf{E}=-\frac{\delta \boldsymbol{B}}{\delta t} ; \nabla \cdot \mathbf{B}=0 ; \nabla \times \mathbf{H}=\mathbf{J}+\frac{\delta \boldsymbol{D}}{\delta t}
$$

Here $\rho(\mathbf{r}, t)$ and $\mathbf{J}(\mathbf{r}, t)$ are the volume density of free charges and current present at the point being considered. If the charges are conserved where $\nabla \cdot \mathbf{J}+\delta \rho / \delta t=0$, then the constitutive relationships which characterize the properties of terrain in terms of permittivity $\varepsilon^{\prime}$, permeability $\mu$ and conductivity $\sigma$ for any given terrain or MUT is given by

$$
\mathbf{D}=\varepsilon^{\prime} \mathbf{E} ; \mathbf{B}=\mu \mathbf{H} ; \mathbf{J}=\sigma \mathbf{E}
$$

If we represent all fields with a time dependence form $\exp (-\mathrm{i} \omega t)$ such that $\mathbf{E}(\mathbf{r})$ is a function of $\mathbf{r}$ only, implying that $\mathbf{E}(\mathbf{r}, t)=\operatorname{Re}\left\{\mathbf{E}(\mathbf{r}) \mathrm{e}^{-\mathrm{i} \omega t}\right\}$ we can substitute eqn (2) into eqn (1) to obtain:

$$
\nabla \cdot \mathbf{E}=\rho / \varepsilon^{\prime} ; \quad \nabla \times \mathbf{E}=\mathrm{i} \omega \mu \mathbf{H} ; \quad \nabla \cdot \mathbf{H}=0 ; \quad \nabla \times \mathbf{H}=\left(\sigma-\mathrm{i} \omega \varepsilon^{\prime}\right) \mathbf{E}
$$

Here $\omega$ is the angular frequency and eqn (3) may be re-written in the form:

$$
\nabla \times \mathbf{H}=-\mathrm{i} \omega \varepsilon_{r} \varepsilon_{0} \mathbf{E}
$$

where

$$
\varepsilon_{r}=\frac{\varepsilon^{\prime}}{\varepsilon_{0}}+\mathrm{i} \frac{\sigma}{\omega \varepsilon_{0}}=\varepsilon_{r}^{\prime}+\mathrm{i} \varepsilon_{r}^{\prime \prime}
$$

is the relative complex dielectric constant of terrain, $\varepsilon_{0}$ is the free space permittivity while $\varepsilon_{r}^{\prime}$ and $\varepsilon^{\prime \prime}{ }_{r}$ are the real and imaginary parts of $\varepsilon_{r}$. The real permittivity, $\varepsilon_{r}^{\prime}$, indicates energy storage by the MUT and the imaginary, $\varepsilon^{\prime \prime}{ }_{r}$ or loss factor indicates how lossy the MUT is to an external field. The $\tan \delta=\varepsilon^{\prime \prime}{ }_{r} \varepsilon_{r}^{\prime}$ is a ratio of energy lost to that stored. Although unused in our approach the conductivity, $\sigma$, is the MUT's ability to conduct electrical current. It is more suitable to liquid MUT; therefore, we use $\varepsilon^{\prime}{ }_{r}$ and $\varepsilon^{\prime \prime}{ }_{r}$ in respect to our approach. 


\subsection{Computer electromagnetic models}

\subsubsection{Monostatic scattering problem}

Different considerations of the general scattering problem have been discussed by several authors including Wei et al. [14] and Ulaby et al. [16]. Here we consider the monostatic configuration of EM transmitter and receiver incident on a multilayer medium where the medium consists of $N+1$ layers separated by $N$ planar sections shown in Fig. 3 .

For such a monostatic configuration, the EM source and the receiver are in the same layer $a$, the transmission medium (air) occupies layer $b$ while the MUT will be in a layer $c$. Subsequent layers may occupy up to $N 1$. If the EM source is in the $z$ direction, then layer $i$ exist between $z_{i-1}$ and $z_{i}$. The real and imaginary dielectric permittivity for each layer is given by $\varepsilon_{r, i}^{\prime}$ and $\varepsilon_{r, i}^{\prime \prime}$, respectively. Therefore, the electrical properties of layers $a, b$ and $c$ are given by the complex permittivities, $\varepsilon_{a}{ }^{*}, \varepsilon_{b}{ }^{*}$ and $\varepsilon_{c}{ }^{*}$. In Wei et al. [14], the transmitter and receiver were in a bistatic formation and not included in the calculation. For simplicity, our approach includes layers $a$ through to layer $c$ and both transmitter and receiver are in a radar monostatic mode. For our models, we consider the magnetic permeability $\mu$ to be constant and both EM transmitter and EM receiver are in the farfield region as expected for radar sensors borne on air or satellite platforms. The farfield distance, $d_{\mathrm{f}}$ is related to the wavelength, $\lambda$, of the EM field being transmitted by an antenna of dimension, $D$ according to:

$$
d_{f}=\frac{2 D^{2}}{\lambda}
$$

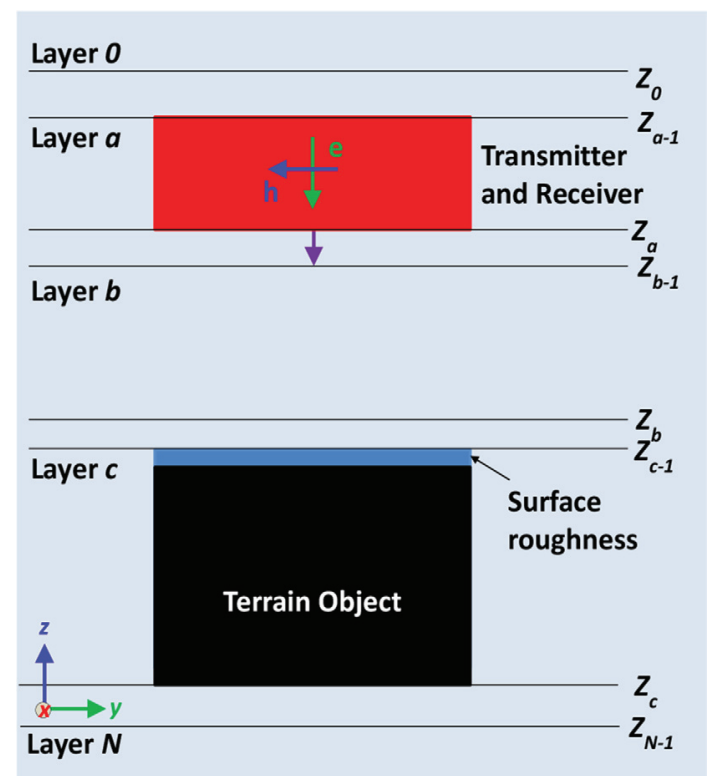

Figure 3: Generic scattering problem with terrain object present in multilayer medium. EM transmitters and receivers are located in layer a while the computational domain (D) comprises layers $a, b$ and $c$. Electrical properties of layer $a, b$ and terrain objectin c are characterized by complex permittivities, $\varepsilon_{a}{ }^{*}, \varepsilon_{b}{ }^{*}$ and $\varepsilon_{c}{ }^{*}$, respectively. 


\subsubsection{Forward modelling}

In the forward problem case, we derive the dielectric properties for input in to our predictive CEM. From eqn (5), we observe that the complex dielectric constant varies with frequency; therefore, we derived measurement values at different frequencies (L-, C- and X-band). The values were input into the CEM models for accuracy and to enhance the models with real world intrinsic terrain data.

For an object in the far field, the total electric field at a point $\mathbf{r}$ which is within the dielectric object terrain will be caused by the transmitter located at position $\mathbf{r}_{\mathrm{T}}$. For monostatic radar configurations, the receiver will be located at position $\mathbf{r}_{\mathrm{R}}$. Based on the superposition principle, the total electric field in the terrain object is a summation of the incident and scattered fields given by [14] as

$$
\mathbf{E}\left(\mathbf{r}, \boldsymbol{r}_{T}\right)=\mathbf{E}^{\mathrm{inc}}\left(\mathbf{r}, \mathbf{r}_{T}\right)+\left(k_{c}^{2}+\nabla \nabla \cdot\right) \int_{D} \mathbf{G}_{c c}^{A J}\left(\mathbf{r}, \mathbf{r}^{\prime}\right) \cdot \chi\left(\mathbf{r}^{\prime}\right) \mathbf{E}\left(\mathbf{r}^{\prime}, \mathbf{r}_{T}\right) \mathrm{d} \mathbf{r}^{\prime}, \mathbf{r} \in D
$$

Where $\mathbf{G}_{c c}^{A J}\left(\mathbf{r}, \mathbf{r}^{\prime}\right)$ is an auxiliary dyadic Green's function, which represents the magnetic vector potential. This equation can also be written with the electric dyadic Green's function $\mathbf{G}_{\mathrm{ac}}^{\mathrm{EJ}}$ at the observation point $\mathbf{r}$ in layer $a$, which is related to the unit current source at a point $\mathbf{r}^{\prime}$ in layer $c$. The wavenumber in layer $c$ is given by $k_{c}^{2}=\omega^{2} \mu_{c} \varepsilon_{c}^{*}$ and $\chi(\mathbf{r})$ is the contrast given by

$$
\chi(\mathbf{r})=\frac{\varepsilon^{*}(\mathbf{r})}{\varepsilon_{c}^{*}}-1
$$

After the total electric field in the computation domain, $D$ is solved using the object equation (i.e. eqn (7)), then the scattered field at any location $S$ can be calculated from:

$$
\mathbf{E}_{a}^{\mathrm{sca}}\left(\mathbf{r}, \boldsymbol{r}_{T}\right)=\mathrm{j} \omega \varepsilon_{c}^{*} \int_{D} \mathbf{G}_{a c}^{E J}\left(\mathbf{r}, \mathbf{r}^{\prime}\right) \cdot \chi\left(\mathbf{r}^{\prime}\right) \mathbf{E}\left(\mathbf{r}^{\prime}, \mathbf{r}_{T}\right) \mathrm{d} \mathbf{r}^{\prime}, \mathbf{r} \in \mathrm{S}
$$

This integral equation that defines the scattered filed at the observation point $S$ is called the data equation.The forward modelling case uses the object and scattered field equations to solve for the scattered field reflected from the terrain object.Several solvers may be used to obtain the solutions of the integral equations. These include full wave solvers such as the method of moments or bi-conjugate gradient method and approximate methods such as the finite element method or the finite integration technique (FIT). Full-wave solvers provide greater accuracy; however, approximate methods can provide good approximations of the exact solution.

In this work, we used FIT implemented by the CST microwave studio (MWS) commercial software tool because it uses less CPU time and memory than full-wave solvers. It also decomposes the computational domain $D$ into a finite number of smaller mesh cells, $d$ in a primary grid $\mathbf{G}$. An internal second or dual mesh $\mathbf{G}$ is set up orthogonally to the primary grid. The CST discretization scheme is shown in Fig. 4.

The Maxwell equations are applied to each mesh cell. This spatial discretization permits flexibility in the geometric attributes such as surface roughness of terrain and the addition of arbitrary material properties such as loss tangent $(\tan \delta)$ dispersion, non-linearity and anisotropy, which is a true reflection of terrain behaviour. In essence, the constitutive equations from eqn (2) become

$$
\mathbf{D}=\boldsymbol{d}_{\varepsilon} \mathbf{E} ; \mathbf{B}=\boldsymbol{d}_{\mu} \boldsymbol{H} ; \mathbf{J}=\boldsymbol{d}_{\sigma} \mathbf{E}
$$




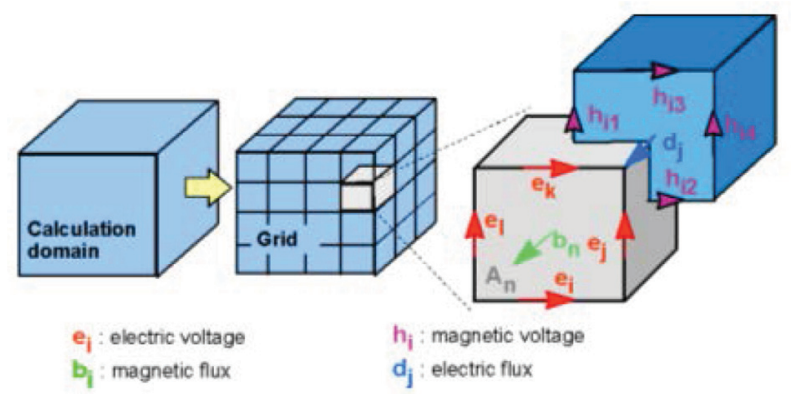

Figure 4: CST implementation of FIT (CST MWS [17]).

In this way, $\boldsymbol{d}_{\varepsilon^{\prime}}, \boldsymbol{d}_{\mu}$ and $\boldsymbol{d}_{\sigma}$ represent the permittivity, permeability and conductivity matrices, respectively. The basic idea of FIT is to approximate the scattered field relative to attributes of the scatterer and the source-dependent diagonal scattering tensor within the grid complexes $\mathbf{G}$ and $\mathbf{G}$. We can evaluate both surface and volume integrals this way. With the transmitter-dependent diagonal scattering tensor, $\Gamma\left(\mathbf{r}, \mathbf{r}_{\mathrm{T}}\right)=\operatorname{diag}\left[\psi_{x}, \psi_{y}, \psi_{z}\right]$, then the scattered field for $\mathbf{r} \in D$ can be written as

$$
\mathbf{E}^{\mathrm{sca}}\left(\mathbf{r}, \mathbf{r}_{T}\right) \approx \Gamma\left(\mathbf{r}, \mathbf{r}_{T}\right) \cdot \mathbf{E}^{\mathrm{inc}}\left(\mathbf{r}, \mathbf{r}_{T}\right) \mathbf{r} \in D
$$

The details of this method can be found in Clemens and Weiland [18] and Weiland [19].

\subsubsection{Model development and simulation}

Surface roughness and geometric properties of the 3D terrain models were developed using uniform spheres based on Graton and Fraser [20]. The radius of each sphere was defined to reflect the aggregate grain size for individual terrain classes such that the intersection of two spheres creates the mean surface roughness at that point. Each terrain model had differing surface roughness and dielectric attributes therefore separate CEMs with different surface roughness implementations were developed for each simulation. The need for an individual CEM per terrain class and combination of terrain and sensor relationships produces specific results. For the electrical characteristics using eqn (10) the measured dielectric permittivity values were input as a matrix across $L-, C$ - and $X$-band frequencies. This way we ensured that the developed 3D EM models accurately represented both physical and electrical properties of each terrain class. Consequently, both homogenous and heterogeneous scattering systems may be represented in this manner. The generic model configuration showing the computational domain, $D$, is shown in Fig. 5.

In order to solve a problem, a finite calculation domain enclosing the terrain problem was created in line with the FIT. Thereafter a mesh system was defined to split the domain in to several grid cells. The general simulation technique used to develop the models with CEM is discussed in [8]. Importantly, the transmitter's angle of incidence, $\theta_{i}$, is varied by mathematical computation to alter the geometry. Our method overcomes the significant challenge of configuring terrain texture and composition while also accurately representing the variation in dielectric permittivity with frequency. 


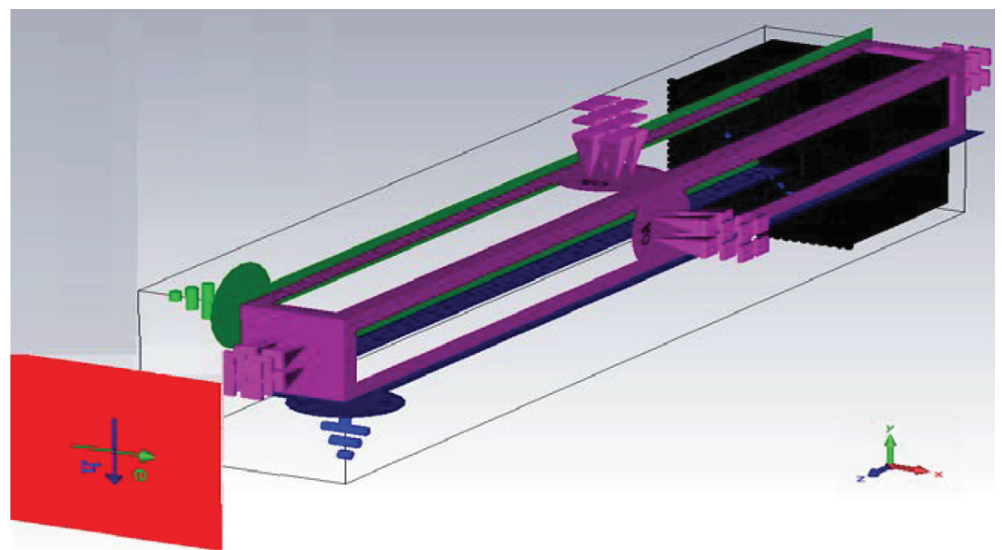

Figure 5: Simulation setup showing linearly polarized incident energy for $\theta_{\mathrm{i}}=90^{\circ}(-Z)$, $0.5 \mathrm{~m}^{3}$ of $3 \mathrm{D}$ terrain $(+Z)$ and open access termination to permit $E^{\text {inc }}$ and $E^{\mathrm{sca}}$ waves to pass through computational domain $D$ with minimum reflection.

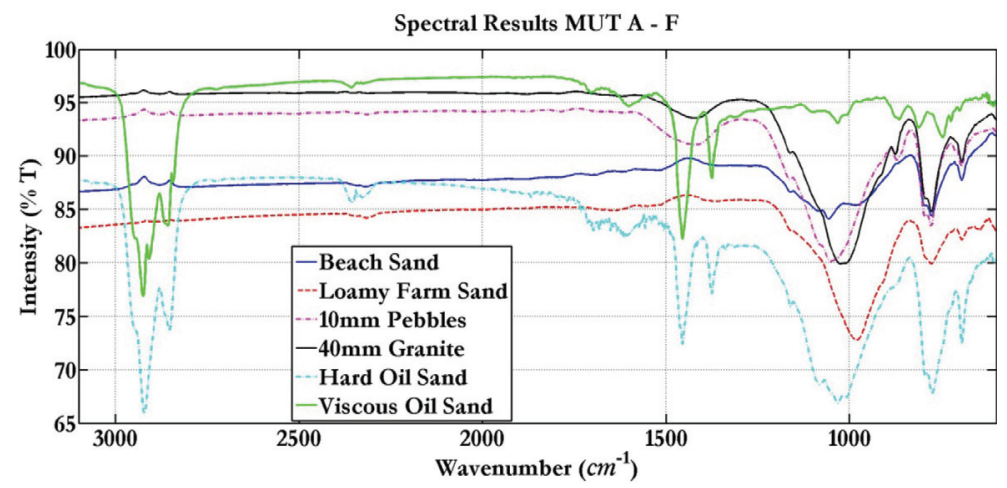

Figure 6: Reflectance spectra $\left(600-3100 \mathrm{~cm}^{-1}\right)$ of six barefaced terrain types.

\section{EXPERIMENTAL RESULTS AND DISCUSSION}

\subsection{Step 1: spectral results}

MUT A-F were spectrally characterized. A zoomed in view of the reflectance spectra is shown in Fig. 6. It covers wavenumber bands, $v=600-3100 \mathrm{~cm}^{-1}$ and intensity scale $40 \%$ $T$ to $110 \% T$. The variation in geochemical signature is due to the variation in electrochemical bonds present. Using our implementation of the SAM classifier to aggregate results and compare with the existing IR solution database, the first thing we observe is the overall emergence of three separate spectral profiles. Above $2000 \mathrm{~cm}^{-1}$, the more homogeneous terrain samples have similar spectral curves. Although the percentage intensity varies, it can be seen that beach sand, 10-mm pebbles and $40-\mathrm{mm}$ gravel/granite produce similar peaks in the $2800-3000 \mathrm{~cm}^{-1}$ region. The heterogeneous terrain produces two distinct profiles. First, both strains of oil sands have a uniquely similar spectral profile with matching similarity in the spectral location of wavenumber bands ( $x$ axis) but with different transmittance peaks 
( $y$ axis) $\% T$. Lastly, the loamy farm soil produces almost no spectral peaks at this region. The bands in this region especially around $3000 \mathrm{~cm}^{-1}$ are indicative of bitumen hydrocarbon due to the exhibition of $\mathrm{C}-\mathrm{H}$ asymmetrical stretching and $\mathrm{C}-\mathrm{H}$ deformation in $-\mathrm{CH}_{3}$ and $-\mathrm{CH}_{2}$ according to Bukka et al. [21].

In the higher wavebands, we detect the presence of clay from the absorption bands between 600 and $1100 \mathrm{~cm}^{-1}$. Hard oil sand and loamy farm sand contain more clay than viscous oil sand and beach sand causing greater absorption features in this region. The granite and pebble MUT have roughly similar amounts of clay causing exactly the same transmittance peaks but a shift in the spectral location of the band due to the particle size. A comparison of all the MUTs is presented in the normalized reflectance spectra shown in Fig. 7. A slight reststrahlen effect is witnessed at $v=1150 \mathrm{~cm}^{-1}$ in beach sand compared with granite, pebbles and loamy farm sand. Wiley [22] considers this is caused by the presence of quartz, which causes an inability of the EM radiation within the narrow energy to propagate within beach sand.

It is clear that for our approach VOS contains more bitumen and less sand particles than HOS although they both have similar geochemical signatures. Furthermore, both 40-mm gravel and 10-mm pebbles share similar geochemical signature with only slight difference in the magnitude of absorption peaks. In addition to the observation of the reststrahlen effect, we believe the homogeneity of beach sand also contributed to the lower absorption profile except in the higher wavenumber region.

\subsection{Step 2: dielectric results}

The choice of dielectric measurement frequency, $f$, will depend on the remote sensing application. For our models, we selected the range of $1-11 \mathrm{GHz}$ to cover the L-, C- and X-band frequencies, which are mostly used for satellite and airborne radar remote sensing of land features. Here we present the dielectric measurement results for the highly homogeneous

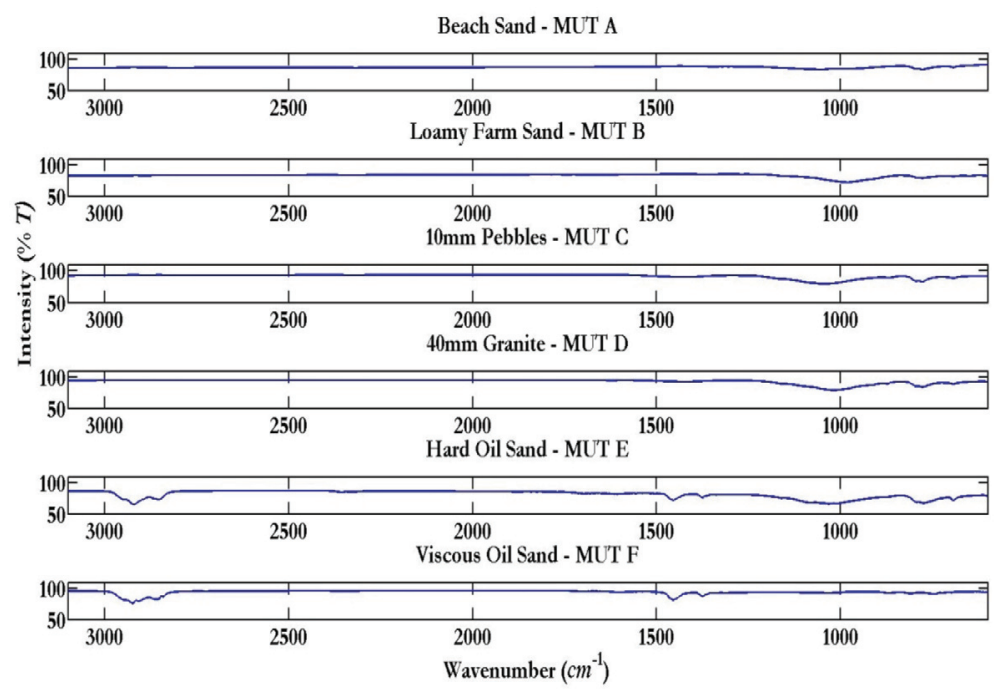

Figure 7: Normalized IR transmittance showing reststrahlen effect at $v=1150 \mathrm{~cm}^{-1}$ for MUT A. 


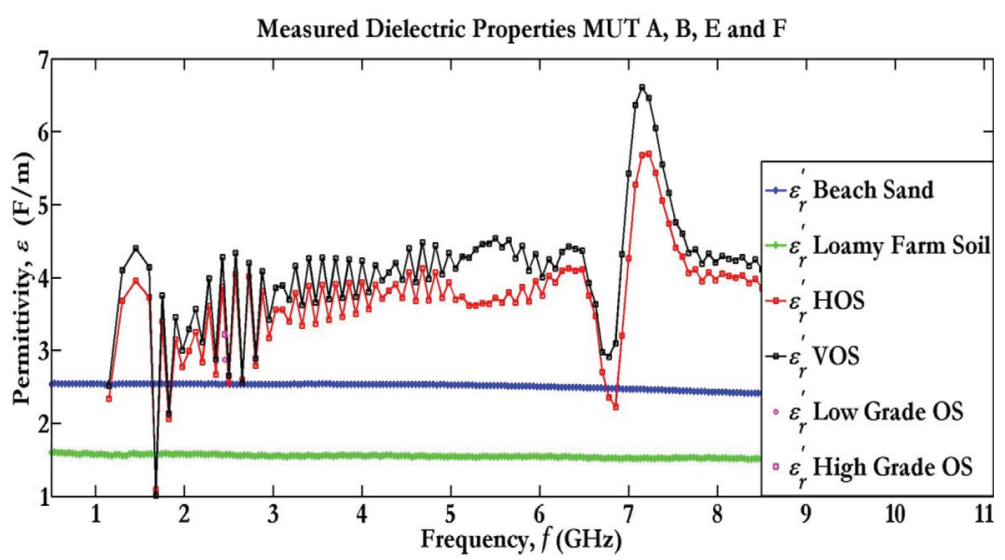

Figure 8: Measured real dielectric permittivity, $\varepsilon_{r}^{\prime}$ of barefaced terrain $(f=0.5-8.5 \mathrm{GHz})$. Results for VOS and HOS compared with low and high grade OS from [24].

terrain (MUT A), mostly homogeneous terrain (MUT B) and the heterogeneous terrain (MUT $\mathrm{E}$ and F) over $0.5-8.5 \mathrm{GHz}$. Due to the presence of sticky bitumen while measuring MUT E and $\mathrm{F}$, care was taken to clean the DPK probe surface with isopropyl alcohol in between measurements as the samples left bitumen residue after each test.

Two key trends emerged in Fig. 8, which shows the measured real dielectric permittivity for barefaced terrain. First, the real permittivity $\varepsilon_{r}^{\prime}$ measurements for MUT A and B were relatively constant with frequency varying between $2.4-2.7 \mathrm{~F} / \mathrm{m}$ and $1.6-1.8 \mathrm{~F} / \mathrm{m}$, respectively. This relative stability of dielectric permittivity for homogeneous terrain has been discussed by Ezeoke and Tong [4] and also by Peake and Oliver [23]. The presence of moisture in beach sand caused the higher permittivity value compared with loamy farm sand. Secondly, the mixture of moisture and bitumen particles in heterogeneous terrain (MUT E and F) caused higher real permittivity $\varepsilon_{r}^{\prime}$ values, greater fluctuation with frequency and more specifically a resonance effect for both HOS and VOS in the upper C-band $(6.5-7.5 \mathrm{GHz})$. In this region, the HOS varied from $2 \mathrm{~F} / \mathrm{m}$ at $6.8 \mathrm{GHz}$ to $5.5 \mathrm{~F} / \mathrm{m}$ at $7.5 \mathrm{GHz}$ while VOS varied from $2.9 \mathrm{~F} / \mathrm{m}$ to $6.8 \mathrm{~F} / \mathrm{m}$ at the corresponding frequency. In essence, the real permittivity fluctuates due to the presence of bitumen.

The imaginary permittivity, $\varepsilon^{\prime \prime}{ }_{r}$ or loss factor data exhibits satisfactory Kramers-Krönig relationship with the real, $\varepsilon_{r}^{\prime}$ permittivity data. This is because the real component of the response at each frequency is related to the behaviour of the imaginary part. In Fig. 9, the $\varepsilon^{\prime \prime}{ }_{r}$ for homogeneous terrain is constant ranging between 0.1 and $0.3 \mathrm{~F} / \mathrm{m}$ but varies for heterogeneous MUT. For HOS, $\varepsilon^{\prime \prime}{ }_{r}$ peaks at $0.5 \mathrm{~F} / \mathrm{m}$ and reaches up to $1.5 \mathrm{~F} / \mathrm{m}$ for VOS at $6 \mathrm{GHz}$. The lowest value for MUT E and MUT F occur at $7 \mathrm{GHz}$ with $3.2 \mathrm{~F} / \mathrm{m}$ for HOS and $3.5 \mathrm{~F} / \mathrm{m}$ for VOS. This means there is a higher tendency for VOS to absorb EM energy in comparison to HOS.The heterogeneous terrain results for both real and imaginary permittivity have been compared with high and low grade oil sand from Erdogan et al. [24]. There was a high correlation (0.96) between both results.

The significance of this step for heterogeneous terrain may be seen from the complex refractive index method (CRIM) for determining the complex dielectric constant in petrophysics. For two phase media, the CRIM formula would expect the real dielectric permittivity $\varepsilon_{r}^{\prime}$ of oil sands to lie midway between the value for wet sand $\left(\varepsilon_{r}^{\prime}=13\right)$ and dry sand $\left(\varepsilon_{r}^{\prime}=2.53\right)$. 


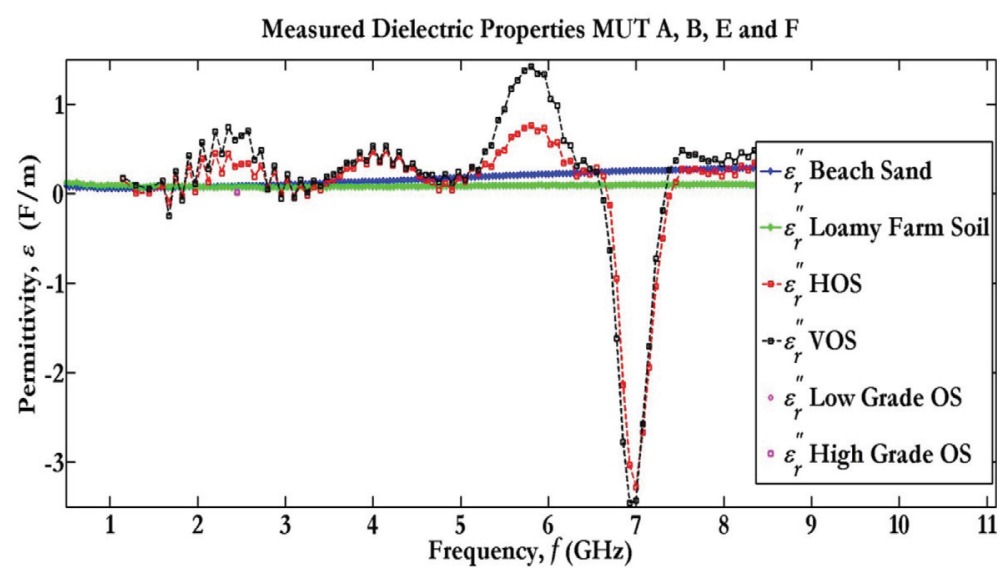

Figure 9: Measured imaginary dielectric permittivity, $\varepsilon^{\prime \prime}{ }_{r}$ of barefaced terrain $(f=0.5-8.5$ $\mathrm{GHz}$ ). Results for VOS and HOS compared with low and high grade OS from [24].

The results in Figs 8 and 9 are affected by the presence of bitumen, which serves to decrease the overall value of $\varepsilon_{c}^{*}$ in the CEM. Consequently, including the measured electrical data in the CEM ensures that the behaviour of the model reflects real life and better permits the polarization mechanisms from spherical grains to be included [8].

\subsection{Step 3: EM signature}

For radar remote sensing, the scattering coefficient $\left(\sigma^{0}\right)$ describes the EM characterization of a target. For a single isolated target with dimensions smaller than the radar system coverage, it is known as the radar cross section (RCS) with the scattered wave at $\mathbf{r}$ :

$$
\mathbf{E}^{\mathrm{sca}}(\mathbf{r})=\mathbf{E}_{0}^{\mathrm{sca}} e^{\mathrm{j} k \mu_{r}}
$$

In situations like terrain imaging where the terrain extends wider than the radar coverage, the target model is represented as an infinite collection of statistical points. The resulting scattered field is due to the coherent addition of the scattered waves from individual scatterers such that:

$$
\mathbf{E}^{\text {sca }}(\mathrm{r})=\sum_{k=1} \mathrm{E}_{k}^{s c a}(\mathrm{r})
$$

In order to express the EM signature or scattering properties of an extended target independently of its area extent as our models do, we describe each elemental terrain target or MUT by the differential RCS or $\mathrm{d} \sigma$. This is the mean RCS over all directions and sometimes referred to as scattering cross section (SCS) because it is for all polarizations [17]. Therefore, the received power, $P_{\mathrm{r}}$, given by the radar equation in $[4,15,16]$ can be considered as the differential power $\mathrm{d} P_{r}$ obtained from an elemental scatterer, $\mathrm{d} s$, so that the radar equation may be written as

$$
\mathrm{d} P_{r}=\frac{P_{t} G_{t}}{4 \pi R_{t}^{2}} \sigma^{0} \mathrm{~d} s \frac{A_{r}}{4 \pi R_{r}^{2}}
$$


Here $P_{t}, G_{t}, R_{t}, R_{r}$ and $A_{r}$ refer to the transmitter power, transmitter gain, transmitter distance, receiver distance and aperture of receiving antenna. The total power received $P_{\mathrm{r}}$ requires integration over the illuminated area, $A_{0}$ to determine the average power returned from the MUT so that eqn (14) becomes

$$
P_{r}=\iint_{A_{0}} \frac{P_{t} G_{t}}{4 \pi R_{t}^{2}} \sigma^{0} \frac{A_{r}}{4 \pi R_{r}^{2}} \mathrm{~d} s
$$

Therefore, the SCS or RCS per unit area (i.e. the scattering coefficient, $\sigma^{0}$ ) is in essence the ratio of statistically averaged scattered power density to the average incident power density over the surface of a sphere with radius $R_{r}$. Mathematically shown as

$$
\sigma^{0}=\frac{\langle\sigma\rangle}{A_{0}}=\frac{4 \pi R_{r}^{2}}{A_{0}} \frac{\left\langle\left|\mathrm{E}^{\mathrm{sca}}\right|^{2}\right\rangle}{\left\langle\left|\mathrm{E}^{\mathrm{inc}}\right|^{2}\right\rangle}
$$

Equation (16) is characterized for a particular frequency along with incident $\left(\theta_{i}, \phi_{i}\right)$ and scattered $\left(\theta_{s}, \phi_{s}\right)$ wave directions. In our case with $M_{T}$ illuminating sources to excite the MUT and $M_{R}$ receivers to collect the scattered field the output consists of data points $M=M_{T} \times M_{R}$ from [14]. In practice, the calculation domain $D$ is discretized into $N$ small cells with linear field quantities and consistent contrast function, $\chi$ in each cell. Therefore, the resultant scattered electric field data $\mathbf{f}$ is a discretized form of eqn (9):

$$
\mathbf{f}\left(\mathbf{r}_{i_{R}}, \mathbf{r}_{i_{T}}\right)=\mathrm{we}_{c}^{*} \sum_{k=1} \mathrm{G}_{a c}^{E J}\left(\mathrm{r}_{i_{R}}, \mathrm{r}_{k}^{\prime}\right) \cdot \mathrm{E}\left(\mathrm{r}_{k}^{\prime}, \mathrm{r}_{i_{T}}\right) \chi\left(\mathrm{r}_{k}^{\prime}\right) \Delta V
$$

So that $\mathbf{f}$ is a $3 M$-dimensional data column with elements that represent the EM signature and $\Delta V$ is the volume element. Similarly, $i_{R}$ and $i_{T}$ represent the receiver and transmitter indices. From [14], $i_{R}=1, \ldots, M_{R}$ and $i_{T}=1, \ldots, M_{T}$.

\section{APPLICATION TO LAND DEGRADATION MONITORING}

The following is an application of our proposed EM signature modelling approach for radar remote sensing to monitor land degradation, which was applied previously for heterogeneous terrain in $[4,8]$ amongst others. Table 1 shows the dielectric permittivity measurement campaign. In [4], we considered the dielectric properties of the MUT to be constant and did not have any knowledge of the geochemical signature. In [8], the FIT method was used for 3D imaging but assumed the MUT is heterogeneous and static.

Thus, in these previous studies, we did not consider homogeneous terrain nor did we reflect the dynamic effect of moisture variation with permittivity on the CEMs. In this work, we

Table 1: Dielectric permittivity measurement campaign.

\begin{tabular}{llll}
\hline MUT & Reference & Description (wt. \%) & Condition \\
\hline Beach sand (BS) & B100 & $100 \%$ BS & Normal \\
& B10W & $10 \%$ water: $90 \%$ BS & At risk \\
& B20W & $20 \%$ water: $80 \%$ BS & Flooding \\
Loamy farm soil (LFM) & LF100 & $100 \%$ LFS & Normal \\
& LF10W & $10 \%$ water: $90 \%$ LFS & At risk \\
& LF20W & $20 \%$ water: $80 \%$ LFS & Flooding \\
\hline
\end{tabular}


included such effects by measuring the dielectric permittivity for two terrain types at three stages representative of flooding conditions measured by the weight percentage of moisture present (wt. \%) in Table 1. A summary of the measured dielectric properties of beach sand and loamy farm soil with different wt. \% composition of moisture as used in the model is shown in Table 2.

The EM transmitter and receiver are located in the farfield region; therefore, the incident EM waves were modelled as plane waves. This monostatic configuration was implemented in the calculation domain $D$. The sensor, terrain and simulation parameters are shown in Table 3. Simulation and postprocessing were performed using CST MWS and Matlab.

The contrast in dielectric permittivity and surface roughness, $\chi$ between the different layers $a, b$ and $c$ of the CEMs enabled the post-processing of the scattering coefficient. A scatter plot of the EM signature comprising the scattered power received, $P_{r}$ for $\theta_{i}=60^{\circ}$ and $30^{\circ}$ is presented in Fig. 10. The EM signature for 'normal', 'at risk' and flooded or degraded terrain varies with incident angle and frequency. For transmit power, $P_{t}$ of $5 \mathrm{~W}$ (r.m.s) incident on terrain the magnitude of received power, $P_{p}$, varies with both frequency and incident angle but does not exceed $0.6 \mathrm{~W}$ (r.m.s) due to propagation path effects and attenuation corresponding to the round trip distance between transmitter, terrain and receiver from eqns (14) and (15).

At low frequency $(1.26 \mathrm{GHz})$, the backscatter received from terrain during normal condition is less than when flooding is about to happen or has already begun regardless of the inclination angle. However, more energy is received by the EM sensor from flooded terrain at $\theta_{i}=60^{\circ}$ than when $\theta_{i}=30^{\circ}$. With increasing frequency, the scattering coefficient decreases because the power returned to the receiver is attenuated with distance. Consequently, less power is detected at $X$-band $(9.7 \mathrm{GHz})$ for both terrain types regardless of the condition. However, during flooding, beach sand with higher permittivity mostly due to increased water content will appear brighter in a radar image for both $L$ - and $X$-band regions compared with farmland due to the higher dielectric permittivity. If the sensor position relative to terrain is

Table 2: Dielectric properties used in the land degradation models.

\begin{tabular}{llllllll}
\hline & & B100 & B10W & B20W & LF100 & LF10W & LF20W \\
\hline$L$ & $\varepsilon_{r}^{\prime}(\mathrm{F} / \mathrm{m})$ & 2.54 & 7.02 & 16.93 & 1.57 & 5.03 & 7.49 \\
& $\operatorname{Tan} \delta$ & 0.0247 & 0.196 & 0.203 & 0.056 & 0.2689 & 0.241 \\
$C$ & ${ }^{\prime}{ }_{r}(\mathrm{~F} / \mathrm{m})$ & 2.52 & 6.22 & 14.719 & 1.55 & 4.22 & 6.50 \\
& $\operatorname{Tan} \delta$ & 0.074 & 0.0854 & 0.150 & 0.0559 & 0.125 & 0.103 \\
$X$ & ${\varepsilon^{\prime}}_{r}(\mathrm{~F} / \mathrm{m})$ & 2.4 & 6.53 & 14.82 & 1.5 & 4.34 & 6.74 \\
& $\operatorname{Tan} \delta$ & 0.1161 & 0.056 & 0.168 & 0.0626 & 0.0878 & 0.056 \\
\hline
\end{tabular}

Table 3: Simulation parameters.

\begin{tabular}{ll}
\hline Parameter & Value \\
\hline Incidence angle $\left(\theta_{i}\right)$ & $90^{\circ}, 60^{\circ}, 30^{\circ}$ \\
Wavelength, $\lambda(\mathrm{cm})$ & $23.5(L), 5.7(C), 3.1(X)$ \\
Resolution cell, $\delta \mathrm{A}$ & $0.5 \mathrm{~m}^{3}$ \\
Sensor position $(\mathrm{m})$ & $5,1,0.5,0,-0.1,-0.5$ \\
\hline
\end{tabular}




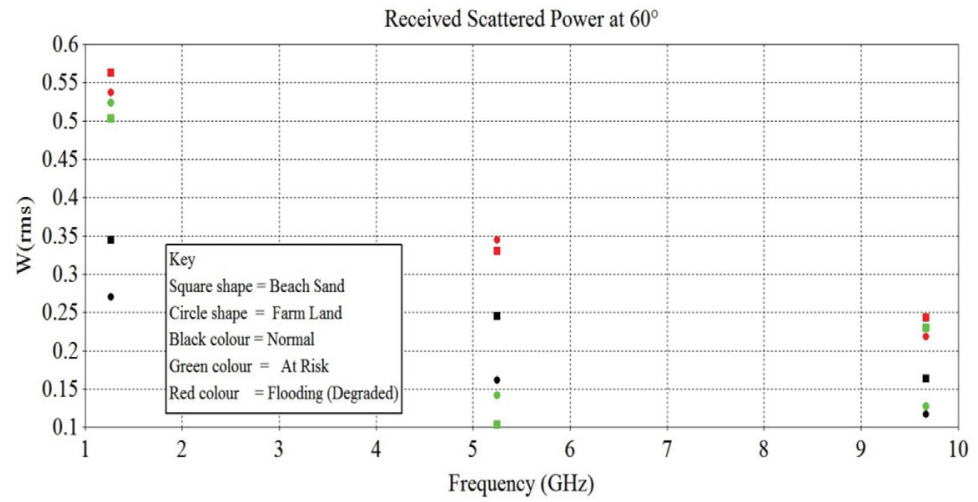

(a)

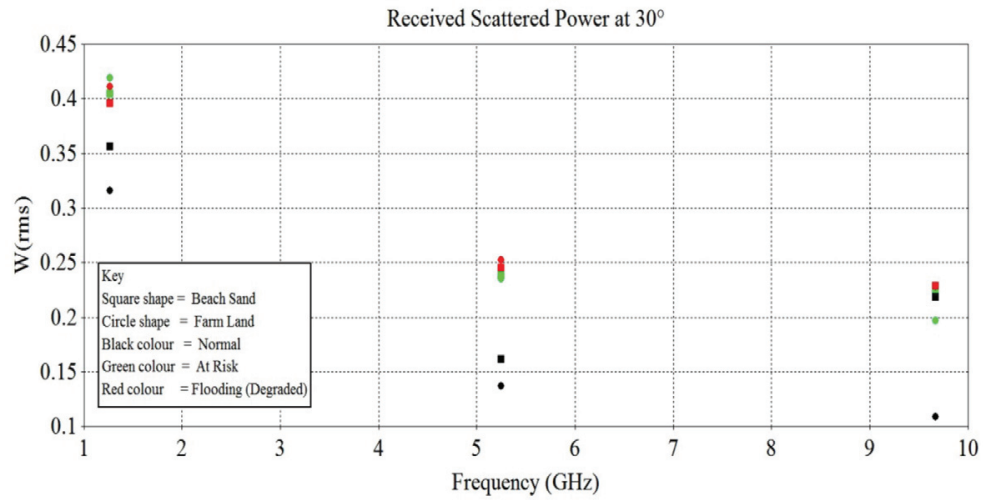

(b)

Figure 10: Scatter plot showing magnitude of scattered power received from homogeneous terrain in normal, at risk and flooding condition when (a) $\theta_{i}=60^{\circ}$ (b) $\theta_{i}=30^{\circ}$ pass through computational domain $D$ with minimum reflection.

altered there is also a change in the backscattered signal. This is why commercial radar satellites acquire data from different 'look angles' and use this to further classify imagery. Importantly, there is greater scattering discrimination for at $\theta_{i}=60^{\circ}$ suggesting that this is a better configuration for monitoring flooding with airborne or spaceborne radar.

\section{CONCLUSION}

An efficient three-step approach for determining the EM signature of homogeneous or heterogeneous barefaced terrain based on geochemical signature, dielectric permittivity and FIT-based CEM has been presented. It has wide spread application to radar remote sensing and was applied to land degradation monitoring before, during and after flooding. In the first step, we empirically investigate the geochemical signature of terrain using FTIR to obtain an approximation of the geochemical properties. Results show that underlying materials such as clay, bitumen and quartz can be distinguished volumetrically. The results are used in the second step to ascertain the dielectric properties, which are also measured. Finally, the dielectric property data are input into the CEM using FIT to obtain the forward model of the scattering coefficient. 
The resilience of the proposed method has been applied to both heterogeneous and homogeneous terrain including an example for land degradation monitoring with a monostatic configuration of transmit and receive antennas at L-, C- and X-band frequencies. Due to the absence of approximations, the approach requires the development of numerous 3D CEMs for each specific combination of sensor and terrain in order to ensure accurate postprocessing of the EM signature. However, we have been able to utilize the contrasts in electrical, physical and chemical properties of the terrain to identify different flooding conditions based on the EM signature. This work is particularly useful to the classification of targets during radar imaging of terrain due to the natural tendency of EM signatures to overlap.

\section{REFERENCES}

[1] Lillesand, T.M., Kiefer, R.W. \& Chipman, J.W., Remote Sensing and Image Interpretation, 5th edn., John Wiley \& Sons: New York, NY, 2004.

[2] Dobson, M.C., Ulaby, F.T. \& Pierce, L.E., Land cover classification and estimation of terrain attributes using synthetic aperture radar. Remote Sensing of Environment, 51, pp. 199-214, 1995. doi: http://dx.doi.org/10.1016/0034-4257(94)00075-x

[3] Ulaby, F.T., SAR biophysical retrievals: "lessons learned and challenges to overcome." Proceedings of the 2nd International Workshop on Retrieval of Bio- and Geo-physical Parameters from SAR Data for Land Applications, 21-23 October, 1998.

[4] Ezeoke, M. \& Tong, K., Synthetic aperture radar signature for oil sands exploration. Proceedings of IEEE UKSim-AMSS 6th European Modeling Symposium (EMS 2012), Valetta, Malta, pp. 419-424, 14-16 November, 2012. doi: http://dx.doi.org/10.1109/ ems.2012.13

[5] Schaber, G.G., SAR studies in the Yuma Desert, Arizona: sand penetration, geology and the detection of military ordnance debris. Remote Sensing of the Environment, 67, pp. 320-347, 1999. doi: http://dx.doi.org/10.1016/s0034-4257(98)00093-5

[6] Ezeoke, M. \& Tong, K., Modeling the electromagnetic reflectivity of Agbabu oil sand from hyperspectral reflectance spectra and dielectric properties at L-, C- and Xband frequencies. IEEE 5th International Conference on Computational Intelligence, Communication Systems and Networks (CICSyN 2013), Madrid, Spain, 5-8 June, 2013. doi: http://dx.doi.org/10.1109/cicsyn.2013.25

[7] Oh, Y., Sarabandi, K. \& Ulaby, F.T., Empirical model \& inversion technique for radar scattering from bare soil surfaces. IEEE Transactions on Geoscience \& Remote Sensing, 30(2), pp. 370-381, 1992. doi: http://dx.doi.org/10.1109/36.134086

[8] Ezeoke, M., Tong, K. \& Shi, S., Modeling synthetic aperture radar signature of agbabu oil sand for petroleum exploration, Energy Production and Management in the 21st Century the Quest for Sustainable Energy, Vol. 2, Eds. C.A. Brebbia, E.R. Magaril and M.Y. Khodorovsky, WIT Transactions on Ecology and the Environment, 190, pp. 1284-1295, 2014. doi: http://dx.doi.org/10.2495/eq141192

[9] Beckmann, P. \& Spizzichino, A., The Scattering of Electromagnetic Waves from Rough Surfaces, Macmillan: New York, NY, 1963.

[10] Torrance, K. \& Sparrow, E., Off-specular peaks in the directional distribution of reflected thermal radiation. ASME Journal of Heat Transfer, 88, pp. 223-230, 1966. doi: http://dx.doi.org/10.1115/1.3691519

[11] Cloutis, E.A., Gaffey, M.J. \& Moslow, T.F., Characterization of minerals in oil sands by reflectance spectroscopy. Fuel, 74(6), pp. 874-879, 1995. doi: http://dx.doi. org/10.1016/0016-2361(95)00016-X 
[12] Xu, D.Q., Ni, G.Q., Jiang, L.L., Shen, Y.T., Li, T., Ge, S.L. \& Shu, X.B., Exploring for natural gas using reflectance spectra of surface soils. Advances in Space Research, 41, pp. 1800-1817, 2008. doi: http://dx.doi.org/10.1016/j.asr.2007.05.073

[13] Yoon, S., Son, J., Lee, W., Lee, H.Y. \& Lee, C.W., Prediction of bitumen content in oil sand based on FT-IR measurement. Journal of Industrial and Engineering Chemistry, 15, pp. 370-374, 2009. doi: http://dx.doi.org/10.1016/j.jiec.2008.11.005

[14] Wei, B., Simsek, E., Yu, C. \& Liu, Q.H., Three-dimensional electromagnetic nonlinear inversion in layered media by hybrid diagonal tensor approximation: stabilized bi-conjugate gradient fast Fourier transform method. Journal of Waves in Random and Complex Media, 17, pp. 129-147, 2007. doi: http://dx.doi.org/10.1080/17455030601016117

[15] Ward, S.H. \& Hohmann, G.W., Electromagnetic theory for geophysical applications (Chapter 4). Electromagnetic Methods in Applied Geophysics: Volume 1, Theory, ed. M.N. Nabighian, Society of Exploration Geophysists: Tulsa, OK, pp. 130-311, 1988.

[16] Ulaby, F.T., Moore, R.K. \& Fung, A.K., Microwave Remote Sensing Active and Passive Vol. 2, Radar Remote Sensing and Surface Scattering and Emission Theory, AddisonWesley: Reading, MA, 1982. doi: http://dx.doi.org/10.1080/01431168408948820

[17] CST Microwave Studio: HF design and analysis tutorial. CST-Computer Simulation Technology, 2012.

[18] Clemens, M. \& Weiland, T., Discrete electromagnetism with the finite integration technique. Progress in Electromagnetics Research, 32, pp. 65-87, 2001. doi: http://dx.doi. org/10.2528/pier00080103

[19] Weiland, T., Time domain electromagnetic field computation with finite difference methods. International Journal of Numerical Modelling: Electronic Networks, Devices and Fields, 9, pp. 295-319, 1996. doi: http://dx.doi.org/10.1002/(sici)10991204(199607)9:4\%3C295::aid-jnm240\%3E3.0.co;2-8

[20] Graton, L.C. \& Fraser, H.J., Systematic packing of spheres with particular relation to porosity and permeability. Journal of Geology, 43, pp. 785-909, 1935. doi: http:// dx.doi.org/10.1086/624386

[21] Bukka, K., Miller, J.D. \& Oblad, A.G., Fractionation and characterization of Utah tarsand bitumen's: influence of chemical composition on bitumen viscosity. Energy \& Fuels, 5(2), pp. 333-340, 1991. doi: http://dx.doi.org/10.1021/ef00026a019

[22] Willey, R.R., Emittance and reflectance of various materials in the 2 to 20 micrometer spectral region. SPIE, 643, pp. 93-100, 1986. doi: http://dx.doi.org/10.1117/12.975521

[23] Peake, W.H. \& Oliver, T.L., The response of terrestrial surfaces at microwave frequencies, Technical Report AFAL-TR-70-301, Ohio State University, Electro-Science Laboratory, Columbus, OH, 279 pp, 1971.

[24] Erdogan, L., Akyel, C. \& Ghannouchi, F.M., Dielectric properties of oil sands at $2.45 \mathrm{GHz}$ with TE1,0,11 mode determined by a rectangular cavity resonator. Journal of Microwave Power and Electromagnetic Energy, 45(1), pp. 15-23, 2007. 